\title{
Genetic Analyses and Mapping of Pink-Root Resistance in Onion
}

\author{
Jen Colcol Marzu' \\ Monsanto Corporation, 1551 Iowa 210, Huxley, IA 50124 \\ Elizabeth Straley \\ Department of Horticulture, University of Wisconsin, 1575 Linden Drive, Madison, WI 53706 \\ Michael J. Havey ${ }^{2}$ \\ USDA-ARS and Department of Horticulture, University of Wisconsin, 1575 Linden Drive, Madison, \\ WI 53706
}

\begin{abstract}
AdDitional INDEX wORDs. disease resistance, quantitative trait locus, single nucleotide polymorphism
Abstract. Pink root [PR (caused by Phoma terrestris)] is a major soil-borne disease of onion (Allium cepa) and reduces both yield and quality of bulbs. PR-resistant cultivars offer the best control option for this disease. The objectives of this study were to complete genetic analyses and mapping of PR resistances from independent sources. Segregating families were developed from different sources of PR resistance and evaluated using a seedling screen. PR severity in two segregating families from the same source of resistance mapped to one position on chromosome 4 with logarithm of odds (LOD) scores of 8.0 and 10.3, and explained $28 \%$ and $35 \%$ of the phenotypic variation, respectively. Estimates of additive and dominance effects revealed this source of $P R$ resistance is codominantly inherited. $P R$ resistance from a second source was assessed by percent survival in the seedling evaluation, showed codominance, and mapped to the same region on chromosome 4 at LOD 12.5 and explained 54\% of the phenotypic variation. This research demonstrates that $\mathrm{PR}$ resistance from different sources mapped to the same chromosome region and showed similar modes of inheritance.
\end{abstract}

Pink root $[\mathrm{PR}$, caused by Phoma terrestris $(\mathrm{Pt})$, synonym Pyrenochaeta terrestris] is a major soil-borne disease of onion (Entwistle, 1990). The disease was first reported in Texas (Tabenhaus, 1917) and has since been identified in onion growing regions worldwide (Chaves and Erickson, 1960; Klingner and Pontis-Videla, 1961; Pfleger and Vaughan, 1972). The characteristic symptom of Pt-infected onion roots is a pink color that progresses to dark purple and eventually the roots disintegrate (Summer, 1995). PR significantly reduces the weight, quality, and yield of onion bulbs (Coleman and Ellerbrock, 1992; Lacy and Roberts, 1982; Perry and Jones, $1955)$ and can become a severe problem especially during seasons of high soil temperatures (Lacy and Roberts, 1982). Methods to control PR include long crop rotations (Entwistle, 1990), soil solarization or fumigation (Ahmed and Harrington, 1974; Hartz et al., 1989; Katan, 1980; Lee et al., 2007; Porter et al., 1989; Rabinowitch et al., 1981), and genetic resistance (Jones and Perry, 1956; Levy and Gornik, 1981; Nichols et al., 1960, 1965).

Numerous studies evaluated onion populations for PR resistance using seedling screens, bulb inoculations, or survival of plants in fields either artificially or naturally infested with Pt. Tabenhaus and Mally (1921) reported that the short-day

\footnotetext{
Received for publication 19 July 2018. Accepted for publication 23 Oct. 2018. We thank Nancy Koval for technical assistance and Karl Broman for statistical advice. We gratefully acknowledge the support of USDA Specialty Crop Research Initiative Block Grant Wisconsin 15-06 and a Monsanto graduate student fellowship for JCM.

Names are necessary to report factually on available data; however, the U.S. Department of Agriculture (USDA) neither guarantees nor warrants the standard of the product, and the use of the name by USDA implies no approval of the product to the exclusion of others that may also be suitable.

${ }^{1}$ These authors contributed equally to this research.

${ }^{2}$ Corresponding author. E-mail: michael.havey@ars.usda.gov.
}

cultivars Crystal Wax and Yellow Bermuda (YB) were resistant to PR. The PR-resistant cultivar Excel 986 was selected from YB and later was used as a parent of other PR-resistant cultivars such as L36, Eclipse (L303), and L365 (Perry and Jones, 1955). Levy and Gornik (1981) screened for resistance to PR using field and greenhouse assays and reported that cultivars Dessex, Granex, Laredo, Grano, and Grano 502 showed PR resistance, while Golden and Yellow Creole were susceptible. In a greenhouse test, Netzer et al. (1985) classified 'Granex' as susceptible to PR, even though the female parent of 'Granex' showed resistance to PR (Perry et al., 1964). Using a seedling test, Nichols (1963) reported low PR infection in 'Excel' and 'Texas Hybrid 28' and high PR infection in 'Texas Grano 502'. PR resistance has also been identified in long-day onions, including cultivars Brigham Yellow Globe, Downing Yellow Globe, and Southport White Globe (Lacy and Roberts, 1982; Nichols, 1963). Using different sources of resistance, Jones and Perry (1956) and Nichols et al. (1965) proposed that PR resistance is conditioned by a single recessive locus.

The goals of this study were to develop segregating families for genetic analyses and mapping of PR resistance in onion. We demonstrate that PR resistances from different sources are conditioned by the same chromosomal region and show codominance.

\section{Materials and Methods}

Seedling evaluation for PR resistance. Segregating families and parents (described below) were evaluated in a seedling screen for PR severity or survival. The isolate of Pt $(T x l c)$ was obtained from PR-infected onion bulbs from Texas and used for all evaluations. The isolate was preserved in sterile soil (Dhingra and Sinclair, 1985), sprinkled onto V8 agar 
Table 1. Most significant single nucleotide polymorphism (SNP), SNPs flanking the 1.5 logarithm of odds (LOD) confidence interval, LOD score, LOD significance threshold at $P=0.05$, additive and dominance effects, and percent phenotypic variation explained for evaluations of pink root severity in segregating families PR1 and PR2 of onion.

\begin{tabular}{lcccccr}
\hline Family & $\begin{array}{c}\text { Most significant } \\
\text { SNP }^{z}\end{array}$ & 1.5 LOD interval & & & & \\
Z & LOD score & LOD threshold & Additive effect & Dominance effect & explained (\%) \\
\hline PR1 & i32829_1057 & i38830_623 to i21519_664 & 8.03 & 3.74 & -2.05 & 0.35 \\
PR2 & i29175_343 & i28612_1057 to i35268_1082 & 10.1 & 4.02 & -2.33 & -0.32 \\
\hline
\end{tabular}

${ }^{\mathrm{z}} \mathrm{SNP}$ markers described by Duangjit et al. (2013); i = isotig.

${ }^{\mathrm{y}}$ Negative additive effect indicates a reduction in pink-root severity conditioned by the resistant (DehyA) parent.

A

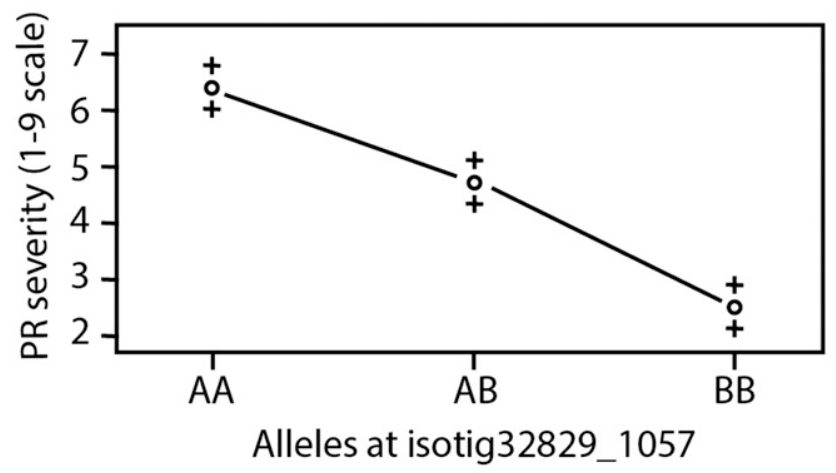

B

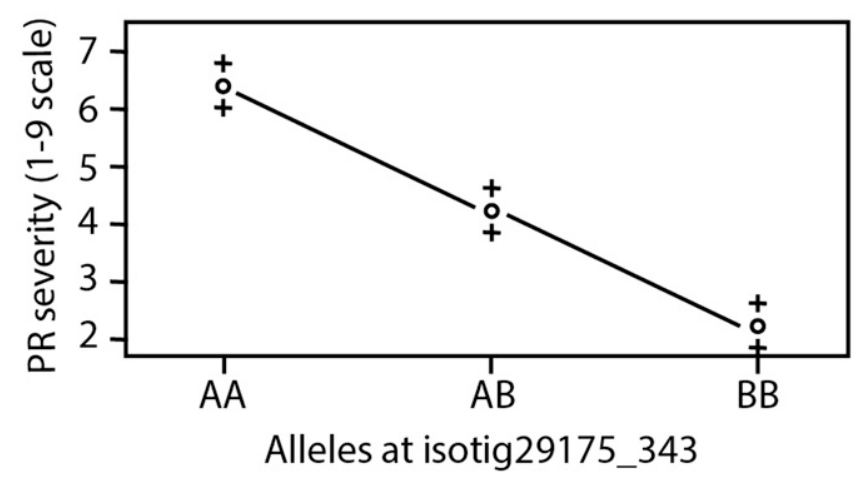

Fig. 1. Additive effects of alleles at isotig32829_1057 (A) and isotig29175_343 (B) on pink-root (PR) severity (y-axis) on a 1 (resistant) to 9 (susceptible) scale for the PR1 (A) and PR2 (B) families of onion, respectively. Allele B from the resistant parent decreased disease severity relative to allele A from the susceptible parent.

plates, and incubated at $24^{\circ} \mathrm{C}$ with 12 -h fluorescent lighting for 7 d. Eight 10-mm-diameter plugs were transferred to a 1000$\mathrm{mL}$ bottle containing $110 \mathrm{~mL}$ of sterile Czapek Dox Broth (Gorenz et al., 1949). The bottles were placed horizontally inside an incubator at $24{ }^{\circ} \mathrm{C}$ with 12 -h fluorescent lighting for $10 \mathrm{~d}$ with gentle mixing every third day. After $10 \mathrm{~d}$ of incubation, the mycelium with broth was chopped for $2 \mathrm{~min}$ in a blender (Jarden Corp., New York, NY), and diluted at a ratio of $100 \mathrm{~mL}$ of inoculum to $400 \mathrm{~mL}$ of non-sterile reverseosmosis (RO) water. This mycelial suspension was mixed with $1000 \mathrm{~mL}$ Hoagland's solution (Sigma, St. Louis, MO) and sprayed into $15 \mathrm{~kg}$ (dry weight) of silica sand [30\% retained on 40 mesh (Industrial Quartz 4030; Unimin Corp., Portage, WI)] in a running concrete mixer. The mixer was allowed to run for 10 min including the spray time. The first batch of infested sand was used to coat the mixer walls and was discarded. Subsequent batches of infested sand were firmly packed into $30 \times 50 \times 10-\mathrm{cm}$ stainless steel pans, and a row template was pressed into the sand to form eight rows divided into two $14-\mathrm{cm}$ sections at a depth of $1.25 \mathrm{~cm}$. Fifty onion seeds were sown per section and covered by pinching sand from each side of the row. Trays were covered with stainless steel lids and placed in water tanks in controlled environment rooms or greenhouses. The temperature was maintained at $19^{\circ} \mathrm{C}$ to allow germination and emergence of onion seedlings with minimal development of the disease. Ten days after sowing (DAS), the temperature was increased to $28^{\circ} \mathrm{C}$ to allow development of PR. Pans were lightly watered along the rows with RO-purified water when the sand was dry and crumbled easily. PR severity was assessed or numbers of survivors were counted at 25 to 34 DAS when the susceptible check (B5351) had $\approx 15 \%$ survival.

DEVELOPMENT AND GENOTYPING OF SEGREGATING FAMILIES. Two segregating families were developed by crossing the malesterile inbred DehyA (mean PR survival of $66.2 \% \pm 12.5 \%$ ) as the female with inbreds B5351C (mean PR survival of $15.2 \% \pm$ $14.8 \%$ ) and Ski-MsMs (mean PR survival of $76.3 \% \pm 11.0 \%$ ). Both of these male parents are homozygous dominant at the nuclear male-fertility restoration $(M s)$ locus and therefore hybrids were male fertile. Single $F_{1}$ plants from each cross were self-pollinated to produce two $\mathrm{F}_{2}$ families (Damon and Havey, 2014). At least 50 random $F_{2}$ progenies from each family were intercrossed in separate cages using flies and seed was bulked from all plants in each cage to produce $\mathrm{F}_{2}$-massed (M) families (PR1 and PR2 for DehyA crossed with B5351C or Ski-MsMs, respectively). Because plants in the PR1 and PR2 families segregated for male fertility, one fourth of plants would be male sterile and not possible to self pollinate to produce progenies for replicated PR evaluations. Therefore, $\mathrm{F}_{2}-\mathrm{M}$ progenies from both families were evaluated for PR severity using the seedling screen. PR severities for 93 and 92 progenies from PR1 and PR2 families, respectively, were scored 25 to 34 DAS when the susceptible parent (B5351C) had $\approx 15 \%$ survival using a 1 to 9 scale, in which 1 = seedling healthy, leaves showing no necrosis; $3=1 \%$ to $25 \%$ of leaves necrotic; $5=$ seedling stunted, $26 \%$ to $50 \%$ of leaves necrotic; $7=$ seedling severely stunted, $51 \%$ to $75 \%$ of leaves necrotic; and $9=$ seedling severely stunted, $76 \%$ to $100 \%$ of leaves necrotic or plant dead (photographs of phenotypes available in Marzu, 2015).

PR-susceptible inbred B5351C was crossed as the female with inbred W446B [mean PR survival of $88.8 \% \pm 15.4 \%$ (Goldman, 1996)]. Groups of three to four hybrid plants were planted together and intercrossed using flies to produce two $\mathrm{F}_{1^{-}}$massed $\left(\mathrm{F}_{1} \mathrm{M}\right)$ families. Plants from these $\mathrm{F}_{1} \mathrm{M}$ families were randomly chosen and self pollinated to produce 54 and $39 \mathrm{~F}_{1}$ massed-selfed ( $\left.\mathrm{F}_{1} \mathrm{MS}\right)$ families (PR3 and PR4, respectively). Four 50-seed replications from each $\mathrm{F}_{1} \mathrm{MS}$ family and parental inbreds were evaluated in a completely randomized design using the seedling screen, and numbers of survivors were counted 25 
Table 2. Most significant single nucleotide polymorphism (SNP), SNPs flanking the 1.5 logarithm of odds (LOD) confidence interval, LOD score, LOD significance threshold at $P=0.05$, additive and dominance effects, and percent phenotypic variation explained for evaluations of seedling survival in the presence of the pink-root fungus for combined analysis of segregating families PR3 and PR4 of onion.

\begin{tabular}{lcccccc}
\hline Most significant SNP & & \multicolumn{2}{c}{ LOD } & & Variation \\
threshold & Additive effect & Dominance effect & explained (\%) \\
\hline i26045_1046 & i38830_623 to i35268_1082 & 12.5 & 3.2 & 23.9 & 1.9 & 54 \\
\hline
\end{tabular}

${ }^{\mathrm{z}} \mathrm{SNP}$ markers were described by Duangjit et al. (2013); $\mathrm{i}=$ isotig.

${ }^{\mathrm{y}}$ Positive additive effect indicates increased \% survival in pink-root evaluations conditioned by the resistant (W446) parent.

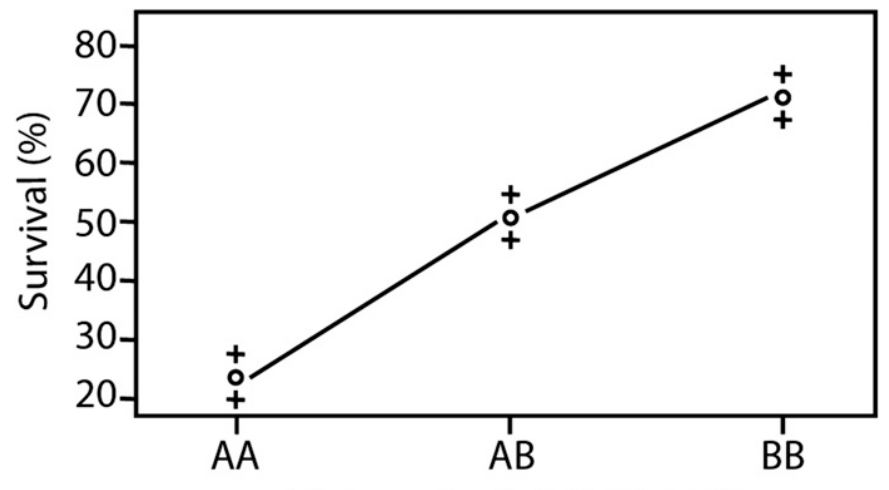

Alleles at isotig26045_1046

Fig. 2. Additive effects of alleles at marker isotig26045_1046 on percent survival of onion seedlings (y-axis) in pink root evaluations from the PR3 and PR4 families. Allele B from the resistant parent increased \% survival relative to allele A from the susceptible parent.

to 34 DAS when the susceptible parent (B5351C) had $\approx 15 \%$ survival. To account for germination differences, 30 seeds from each family were planted in the greenhouse and resulting plants counted 30 DAS to estimate the germination rate. The mean percent PR survival was adjusted by the respective germination rate for each family.

DNA was isolated using a mini-preparation (Nucleospin 96 Plant II; Macherey-Nagel, Bethlehem, PA) from 93 and 92 segregating $\mathrm{F}_{2} \mathrm{M}$ plants from the PR1 and PR2 families and from the 54 and $39 \mathrm{~F}_{1} \mathrm{M}$ plants used to produce the PR3 and PR4 families, respectively. DNA quantities were determined using a spectrophotometer (Nanodrop ND-1000; Thermo Fisher Scientific, Waltham, MA) and qualities were assessed by electrophoresis through $1 \%$ agarose gels. For the $\mathrm{F}_{2} \mathrm{M}$ progenies from the PR1 and PR2 families, insufficient DNA was isolated from seedlings for genotyping and a whole-genome amplification (LGC Genomics, Boston, MA) was used to increase DNA quantity. For the PR1 and PR2 families, 395 single nucleotide polymorphisms (SNP) spread across the eight chromosomes of onion were genotyped using the KASPar assay (Duangjit et al., 2013). For the PR3 and PR4 families, 169 SNPs (Duangjit et al., 2013) and two SNP markers reported as linked to $P R$ resistance in a patent application (Black et al., 2015) were genotyped using the KASPar assay. Segregations from the PR3 and PR4 families were combined after establishing homogeneity of errors (Gomez and Gomez, 1984). Goodness-of-fit to the expected 1:2:1 ratio and genetic mapping as $F_{2}$ families were completed using Joinmap 4.0 and the Kosambi mapping function (Van Ooijen, 2006). Genetic distances in centimorgans will be larger for the segregating families used in this research than for $\mathrm{F}_{2}$ families due to the two generations of recombination.

Quantitative Trait ANAlyses. For the PR1 and PR2 families, severity scores were not normally distributed and $\log$ and square-root transformations were calculated. R/qtl (Broman et al., 2003; R Core Team, 2013) was used for composite interval mapping (CIM) to identify quantitative trait loci (QTL) conferring PR resistance, and two covariates and a 10-cM window size were used in the CIM mapping. For the PR3 and PR4 families, the Shapiro-Wilk test confirmed a normal distribution of the survival data and analysis used imputation and forward/backward selection in the R/qtl (Broman et al., 2003) and R/broman (Broman and Broman, 2016) packages in R Studio V1.0.136 (R Studio Team, 2016). Multiple imputation was used because not all SNPs segregated in both the PR3 and PR4 families, resulting in missing data, and this approach is better with missing scores due to monomorphic markers. For all analyses, 1000 permutations were completed to determine the 0.05 significance logarithm of odds (LOD) threshold. After identifying a QTL, the maximum LOD score, additive and dominance effects, and percent phenotypic variation explained by the QTL were calculated. The most significant SNP and loci flanking the 1.5 LOD intervals were identified.

\section{Results and Discussion}

The PR1 and PR2 segregating families were developed from crosses of a seed parent (DehyA) with an intermediate level of PR resistance (mean PR survival of $66.2 \% \pm 12.5 \%$ ) with B5351 (mean PR survival of $15.2 \% \pm 14.8 \%$ ) and Ski-MSMS (mean PR survival of $76.3 \% \pm 11.0 \%$ ) male parents, respectively. Both families segregated for PR resistance and were not significantly different for mean survival at $50.0 \% \pm 22.6 \%$ and $50.0 \% \pm 19.5 \%$, respectively. Although the cross of DehyA and Ski-MSMS was between two inbred lines showing PR resistance, one of the plants used for crossing must have been susceptible to PR and the PR2 family segregated for resistance. Both the PR1 and PR2 families were evaluated using the seedling screen and individual progenies scored for PR severity on the 1 to 9 scale. A total of 111 SNPs were mapped using 93 $\mathrm{F}_{2} \mathrm{M}$ progenies from the PR1 family and $162 \mathrm{SNPs}$ with $92 \mathrm{~F}_{2} \mathrm{M}$ progenies from the PR2 family (SNPs and segregations reported in Marzu, 2015). Using non-transformed severity scores, PR resistance in both families mapped to the same position on chromosome 4 at LOD scores of 8.0 and 10.1 and explained $28 \%$ and $35 \%$ of the phenotypic variation for PR1 and PR2 families, respectively (Table 1). The same chromosome region was detected using $\log$ and square-root transformations of severity scores. Additive effects in both families were larger than dominance effects (Table 1), and in both families PR resistance from the DehyA parent decreased disease severity and was codominantly inherited (Fig. 1).

For the PR3 and PR4 families, 54 and $39 \mathrm{~F}_{1} \mathrm{MS}$ families, respectively, were screened in replication for percent survival. After establishing homogeneity of errors, 151 SNPs were used for mapping of PR survival in these two families (SNPs and segregations reported in Straley, 2018). One highly significant 
Table 3. Sequences of single nucleotide polymorphisms (SNP) on chromosome 4 of onion mapping within the 1.5 logarithm of odds (LOD) confidence interval for pink root-resistance in three segregating families.

\begin{tabular}{ll}
\hline SNP & \multicolumn{1}{c}{ Sequence flanking SNP } \\
\hline isotig21519_664 & TGATGTATCGTACCGTGCTCTTGAANTTTCATCAGAANGCACCTCATAGGCCAGACGAAT[A/G] \\
NNCTTGAATACCTCACTGGCCTGTGAATCTTGACTGACATCAGGATGNTANTTTCTAGCA & GGCGCAAGGCCTTGCAGTGGGTTCTTCCCTTGTGGAAGAAGACAAGCTAGAGCTTGCCAC[A/G] \\
isotig26045_1046 & TCTCTTCTTGCGAAAGCCAAAGCTAAAGGTGTTTCTTTGTTGCTTCCCACTGATGTTATT \\
isotig29175_343 & GTATATGGAGACTGGAAAGCTTCTAGCAGTTGTTCAGTCAGGTGGAGAGTTTGTTACCAA[C/T] \\
isotig32829_1057 & AAAGATGGAACAATATCTTATAGTGGTGGTGAAGCACATGCTCTTGATATTGGCCGTGAT \\
isotig33399_1211 & AATCGCCGCTTCCACTTCCCATATTTTGCAAAACACTCCCTCCACTACCTGCATTCTGCA \\
isotig35268_1082 & TCTATCAGCTGGATGATTAAACCTGCATGTCATTCCAAACCTGCAAATCCCCGTGCGCAT[A/G] \\
isotig38830_623 & TAATATGAGCAGTCTGGCTCGCCAGGCCTCTCTGGATATTGCCCACTTCCCATATCTTCG \\
& CTTAGGAGGTCAGCTGATACTCTTTGGCAGATCCAGCAGCAGCTTGATCTGTAATCAGTT[C/T] \\
& TNAACTTTTTGAGTTATTGTACGGACACATATTGTGTTTTGTCGTNCNNNNNNNNNNNNN \\
& TTTGGACAACCTCAACTTCTTCCTACTTCCATCNCCATCTTCTTCATCGCTATTATTACT[A/G] \\
& NNGTTACTTTCTCCAGTGCTTCTTTTTCCACTTNGCAAGCTTGTAATCGTACTGTGTGGA
\end{tabular}

${ }^{\mathrm{z}}$ Sequences were previously reported by Duangjit et al. (2013) and are listed here for convenience only. SNP shown in brackets. N indicates nucleotides not scored due to low confidence.

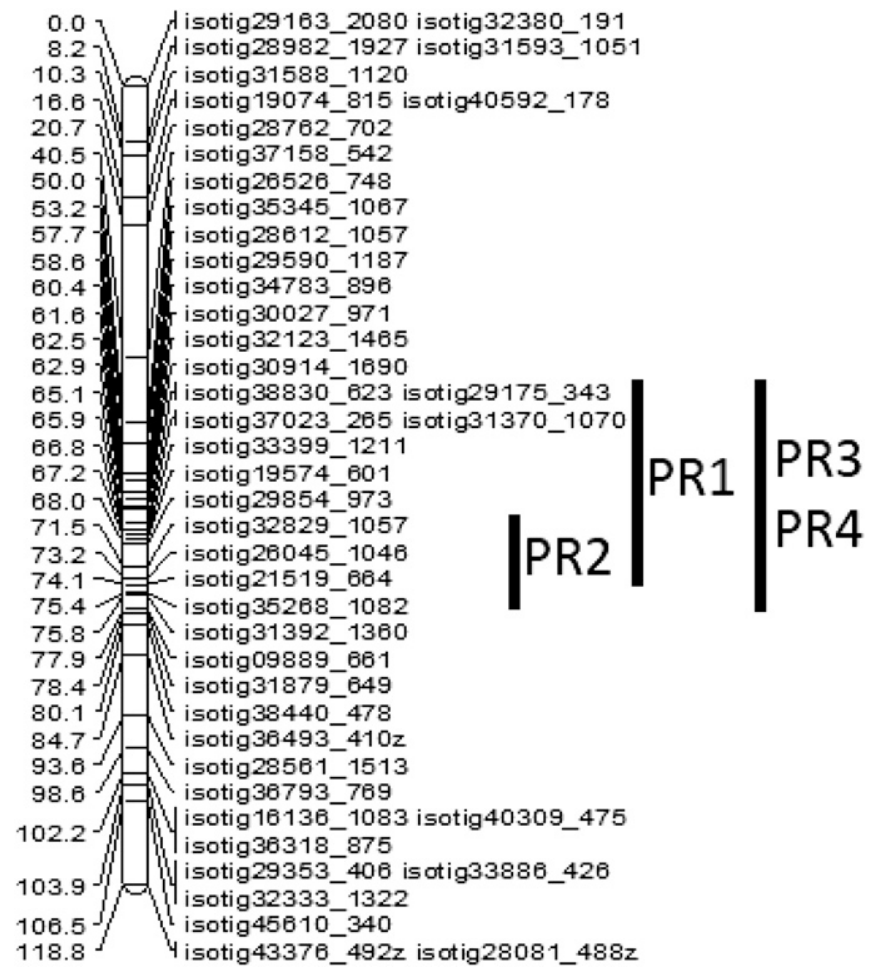

Fig. 3. Genetic linkages on chromosome 4 of onion among single nucleotide polymorphisms (SNPs) across the 1.5 logarithm of odds (LOD) confidence interval (black bars) for pink-root resistance for segregating families PR1 $($ DehyA $\times$ B5351), PR2 (DehyA $\times$ Ski-MsMs), and the combined analysis of PR3 and PR4 (B5351 $\times$ W446) families. SNP names are shown on right (Duangjit et al., 2013) and genetic distances in centimorgans are shown on left. Genetic map was developed using an $\mathrm{F}_{2}$ family from DehyA $\times$ B5351 as reported by Damon and Havey (2014).

QTL explained 54\% of the phenotypic variability for PR survival (Table 2) and mapped to the same region on chromosome 4 as the PR1 and PR2 families. The PR resistance QTL was located closest to SNP marker isotig26045_1046, and the positive additive effect of the region tagged by marker isotig26045_1046 indicated that for every W446B allele added, PR survival increased by $24 \%$ (Fig. 2).
We attempted to join the genetic maps from the PR1, PR2, PR3, and PR4 families, but too few SNPs segregated across families to construct a consensus map. However SNPs within the 1.5 LOD confidence intervals for the PR1, PR2, and PR3/ PR4 families (Table 3) were previously mapped by Damon and Havey (2014) using a segregating $F_{2}$ family from the same parents as the PR1 family, and positions of the 1.5 LOD confidence intervals are shown in Fig. 3. In a patent application, Black et al. (2015) mapped PR resistance from the short-day population SYG-75-1706 and reported that resistance showed a significant additive effect of $32 \%$ increased seedling survival. We determined the segregations of two SNP markers (NQ0257962 and NQ0257570) reported in this patent application as linked to PR resistance, and both mapped within the 1.5 LOD interval on chromosome 4 for PR resistance in the PR3 and PR4 families. NQ0257962 mapped between SNPs isotig26045_1046 and isotig38830_623 and NQ0257570 between SNPs isotig35268_1082 and isotig33399_1211. The results of our study and that of Black et al. (2015) reveal that three sources of PR resistance show codominance in seedling evaluations and map to the same region on chromosome 4 (Fig. 3). Earlier studies evaluated segregating families in Pt-infested field plots (Jones and Perry, 1956) and in a seedling assay (Nichols et al., 1965) similar to the one used in this study, and both concluded that PR resistance is recessively inherited. These recessive PR resistances may represent different resistance mechanism(s) to PR. The SNPs identified in this study (Table 3) associated with PR resistance will be useful for comparative mapping of different sources of resistance, and allow independent resistances to be combined with the codominant source identified by this study to potentially increase overall PR resistance. The SNP markers identified in this study will also be useful for the introgression of the PR resistance on chromosome 4 into diverse onion populations.

\section{Literature Cited}

Ahmed, A.A. and J.F. Harrington. 1974. Onion seed yield as affected by pink rot disease, soil fumigation, mother bulb fertilization and bulb size. HortScience 4:394-396.

Black, L., E.K.F. Chan, J.F. Colcol, R. Jones, C. Kramer, and W. Xiang. 2015. Disease resistance loci in onion. United States patent application 20150150155A1. Filed 28 May 2015. 
Broman, K.W. and A.T. Broman. 2016. R/broman: Karl Broman's R Code. R package version 0.62-1. 28 Aug. 2018. <https://CRAN.Rproject.org/package $=$ broman $>$.

Broman, K.W., H. Wu, S. Sen, and G.A. Churchill. 2003. R/qt1: QTL mapping in experimental crosses. Bioinformatics 19:889-890.

Chaves, G.M. and H.T. Erickson. 1960. Pink root disease on onions in

Minas Gerais, Brazil. Plant Dis. Rpt. 44:877.

Coleman, P.M. and L.A. Ellerbrock. 1992. Interaction of Phoma terrestris and soil moisture level on yield of two onion cultivars differentially susceptible to pink root. Plant Dis. 76:1213-1216.

Damon, S.J. and M.J. Havey. 2014. Quantitative trait loci controlling amounts and types of epicuticular waxes in onion. J. Amer. Soc. Hort. Sci. 139:597-602.

Dhingra, O.D. and J.B. Sinclair. 1985. Basic plant pathology methods. 2nd ed. CRC Press, Boca Raton, FL.

Duangjit, J., B. Bohanec, A.P. Chan, C.D. Town, and M.J. Havey. 2013. Transcriptome sequencing to produce SNP-based genetic maps of onion. Theor. Appl. Genet. 126:2093-2101.

Entwistle, A.R. 1990. Root diseases, p. 103-154. In: H.D. Rabinowitch and J.L. Brewster (eds.). Onion and allied crops. Vol II. CRC Press, Boca Raton, FL.

Goldman, I.L. 1996. A list of germplasm releases from the University of Wisconsin onion breeding program, 1957-1993. HortScience 31:878-879.

Gomez, K.A. and A.A. Gomez. 1984. Statistical procedures for agricultural research. Wiley, New York, NY.

Gorenz, A.M., R.H. Larson, and J.C. Walker. 1949. Factors affecting pathogenicity of pink root fungus of onions. J. Agr. Res. 78:1-18.

Hartz, T.K., C.R. Bogle, D.A. Bender, and F.A. Avila. 1989. Control of pink root disease in onion using solarization and fumigation. J. Amer. Soc. Hort. Sci. 114:587-590.

Jones, H.A. and B.A. Perry. 1956. Inheritance of resistance to pink root in the onion. J. Hered. 47:33-34.

Katan, J. 1980. Solar pasteurization of soils for disease control: Status and prospects. Plant Dis. 64:450-454.

Klingner, A. and R.E. Pontis-Videla. 1961. Pink root disease of onions in Mendoza, Argentina. Plant Dis. Rpt. 45:235.

Lacy, M.L. and D.L. Roberts. 1982. Yields of onion cultivars in Midwestern organic soils infested with Fusarium oxysporum f. sp. cepae and Pyrenochaeta terrestris. Plant Dis. 66:10031006.

Lee, C.J., J.T. Lee, J.S. Moon, L.I. Ha, H.D. Kim, W. Kim, and M. G. Cheon. 2007. Effects of solar heating for control of pink root and other soil-borne diseases of onions. Plant Pathol. 23:295299.
Levy, D. and A. Gornik. 1981. Tolerance of onions (Allium cepa L.) to the pink root disease caused by Pyrenochaeta terrestris. Phytoparasitica 9:51-57.

Marzu, J.C. 2015. Genetic analyses of resistances to Fusarium basal rot and pink root in onion. PhD Diss., Univ. Wisconsin, Madison.

Netzer, D., H.D. Rabinowitch, and C.H. Weintal. 1985. Greenhouse technique to evaluate onion resistance to pink root. Euphytica 34:385-391.

Nichols, C.G. 1963. Determination of the inheritance of resistance in the onion, Allium cepa L. to the pink root fungus Pyrenochaeta terrestris. PhD Diss., Univ. Wisconsin, Madison.

Nichols, C.G., R.H. Larson, and W.H. Gabelman. 1960. Relative pink root resistance of commercial onion hybrids and varieties. Proc. Amer. Soc. Hortic. Sci. 76:468-469.

Nichols, C.G., W.H. Gabelman, R.H. Larson, and J.C. Walker. 1965. The expression and inheritance of resistance to pink root in onion seedlings. Phytopathology 55:752-756.

Perry, B.A. and H.A. Jones. 1955. Performance of short-day pink-rootresistant varieties of onions in southern Texas. J. Amer. Soc. Hort. Sci. 66:350-353.

Perry, B.A., E.W. Davis, and H.A. Jones. 1964. Establishment of a pink root nursery. Econ. Bot. 18:77-79.

Pfleger, F.L. and E.K. Vaughan. 1972. Pyrenochaeta terrestris as a naturally occurring component of the soil in Oregon. Plant Dis. Rep. 56:180-182.

Porter, I.J., P.R. Merriman, and P.J. Keane. 1989. Integrated control of pink root (Pyrenochaeta terrestris) of onions by dazomet and soil solarization. Austral. J. Agr. Res. 40:861-869.

R Core Team. 2013. R: A language and environment for statistical computing. 28 Aug. 2018. <http://www.R-project.org/>.

R Studio Team. 2016. R Studio: Integrated development for R. R Studio, Boston, MA.

Rabinowitch, H.D., J. Katan, and I. Rotem. 1981. The response of onions to solar heating, agricultural practices and pink-root disease. Scientia Hort. 15:331-340.

Straley, E. 2018. QTL analyses of Fusarium basal rot and pink root resistance in onion. MS Thesis., Univ. Wisconsin, Madison.

Summer, D.R. 1995. Pink root, p. 12-13. In: H.F. Schwartz and S.K. Mohan (eds.). Compendium of onion and garlic diseases. APS Press, St. Paul, MN.

Tabenhaus, J.J. 1917. Pink root, a new disease of onions in Texas. Phytopathology 7:217 (abstr.).

Tabenhaus, J.J. and F.W. Mally. 1921. Pink root disease of onions and its control in Texas. Texas Agr. Exp. Sta. Bul. 273.

Van Ooijen, J.W. 2006. JoinMap®4, Software for the calculation of genetic linkage maps in experimental populations. Kyazma, Wageningen, Neth. 